\title{
Intranasal carbetocin reduces hyperphagia in individuals with Prader-Willi syndrome
}

\author{
Elisabeth M. Dykens, ${ }^{1}$ Jennifer Miller, ${ }^{2}$ Moris Angulo, ${ }^{3}$ Elizabeth Roof, ${ }^{1}$ Michael Reidy, ${ }^{4}$ \\ Hind T. Hatoum, ${ }^{5}$ Richard Willey, ${ }^{4}$ Guy Bolton, ${ }^{4}$ and Paul Korner ${ }^{4}$ \\ 'Kennedy Center for Research on Human Development, Vanderbilt University, Nashville, Tennessee, USA. \\ ${ }^{2}$ Division of Pediatric Endocrinology, University of Florida, College of Medicine, Gainesville, Florida, USA \\ ${ }^{3}$ Department of Pediatrics, Winthrop University Hospital, Mineola, New York, USA. ${ }^{4}$ Ferring Pharmaceuticals Inc., \\ Parsippany, New Jersey, USA. ${ }^{5}$ Hind T. Hatoum \& Company, Chicago, Illinois, USA.
}

Conflict of interest: JM has received research funding from Ferring, Zafgen, and Rhythm Pharmaceuticals. MR is a salaried employee of Ferring and is listed as coinventor on a patent application related to the content of this manuscript, with no financial claims thereof (patent no. W0 2016044131 A1). HTH is the president of Hind T. Hatoum \& Company, which had a contractual agreement with Ferring International Pharmascience Center US Inc., the developer of an investigational drug that is the subject of the present manuscript, that involved strategies and the performance of some of the analyses related to data presented in the manuscript. PK was a salaried employee of Ferring and is listed as coinventor on a patent application related to the content of this manuscript, with no financial claims thereof (patent no. WO 2016044131 A1)

Submitted: November 7, 2017

Accepted: May 4, 2018

Published: June 21, 2018

\section{Reference information:}

JCI Insight. 2018;3(12):e98333. https://doi.org/10.1172/jici.

insight.98333.
BACKGROUND. Prader-Willi syndrome (PWS) is a genetic neurodevelopmental disorder of lifethreatening hyperphagia, obesity, intellectual deficits, compulsivity, and other behavioral problems. The efficacy and safety of i.n. carbetocin, an oxytocin analog, was evaluated in a prospective, randomized, double-blinded trial in adolescents with PWS.

METHODS. Eligible patients aged $10-18$ years with genetically confirmed PWS were randomized (1:1) to i.n. carbetocin or placebo 3 times daily for 14 days. The primary efficacy endpoint was change in parent/caregiver-rated Hyperphagia in PWS Questionnaire-Responsiveness (HPWSQ-R) total score. Secondary efficacy endpoints included HPWSQ-R behavior, drive, and severity domains; clinician-rated HPWSQ; Children's Yale-Brown Obsessive-Compulsive Severity Scale; food domain of the Reiss Profile; and Clinical Global Impression-Improvement scale. Endpoints were assessed using analysis of covariance. Relationship between primary and secondary endpoints was assessed using Pearson correlation coefficients. Safety was assessed throughout the study.

RESULTS. Demographics and clinical characteristics were similar between treatment groups (carbetocin, $n=17$; placebo, $n=20$ ). Patients receiving carbetocin had statistically significant reductions in HPWSQ-R total score at study end $(-15.6)$ versus patients receiving placebo $(-8.9 ; P=$ 0.029); several secondary efficacy endpoints also demonstrated significant differences $(P<0.05)$. Treatment effects for the primary and secondary endpoints were highly correlated $(P \leq 0.0001)$. Incidence of adverse events (AEs) was similar between treatment groups.

CONCLUSION. I.n. carbetocin was well tolerated and improved hyperphagia and behavioral symptoms of PWS.

TRIAL REGISTRATION. ClinicalTrials.gov: NCT01968187

FUNDING. The study was funded by Ferring Pharmaceuticals. Recruitment was aided by ongoing work in PWS performed through Eunice Kennedy Shriver National Institute of Child Health and Human Development grant U54 HD083211.

\section{Introduction}

Prader-Willi syndrome (PWS) is a complex, multisystem, neurodevelopmental disorder occurring in approximately 1 in 15,000 live births $(1,2)$. The underlying cause of PWS is the lack of expression of paternally inherited imprinted genes on chromosome 15q11-q13, most commonly through paternal deletions or maternal uniparental disomy $(3,4)$.

These genetic anomalies lead to a distinctive phenotype that includes mild to moderate levels of intellectual disability, compulsivity, growth hormone deficiency, life-threatening hyperphagia, and high risk of obesity.

Symptoms of PWS often present as a period of infantile hypotonia and feeding difficulties that gradually improve, followed by an increased interest in food in early childhood (age 4-5 years) (5). Hyperphagia and food-related behavior problems often evolve by 8 years of age and are characterized by the lack of a normal satiety response, food preoccupations, and problematic food-seeking behaviors. Although symptom severity varies, hyperphagia poses persistent, life-long risks to the health and safety of affected individuals 


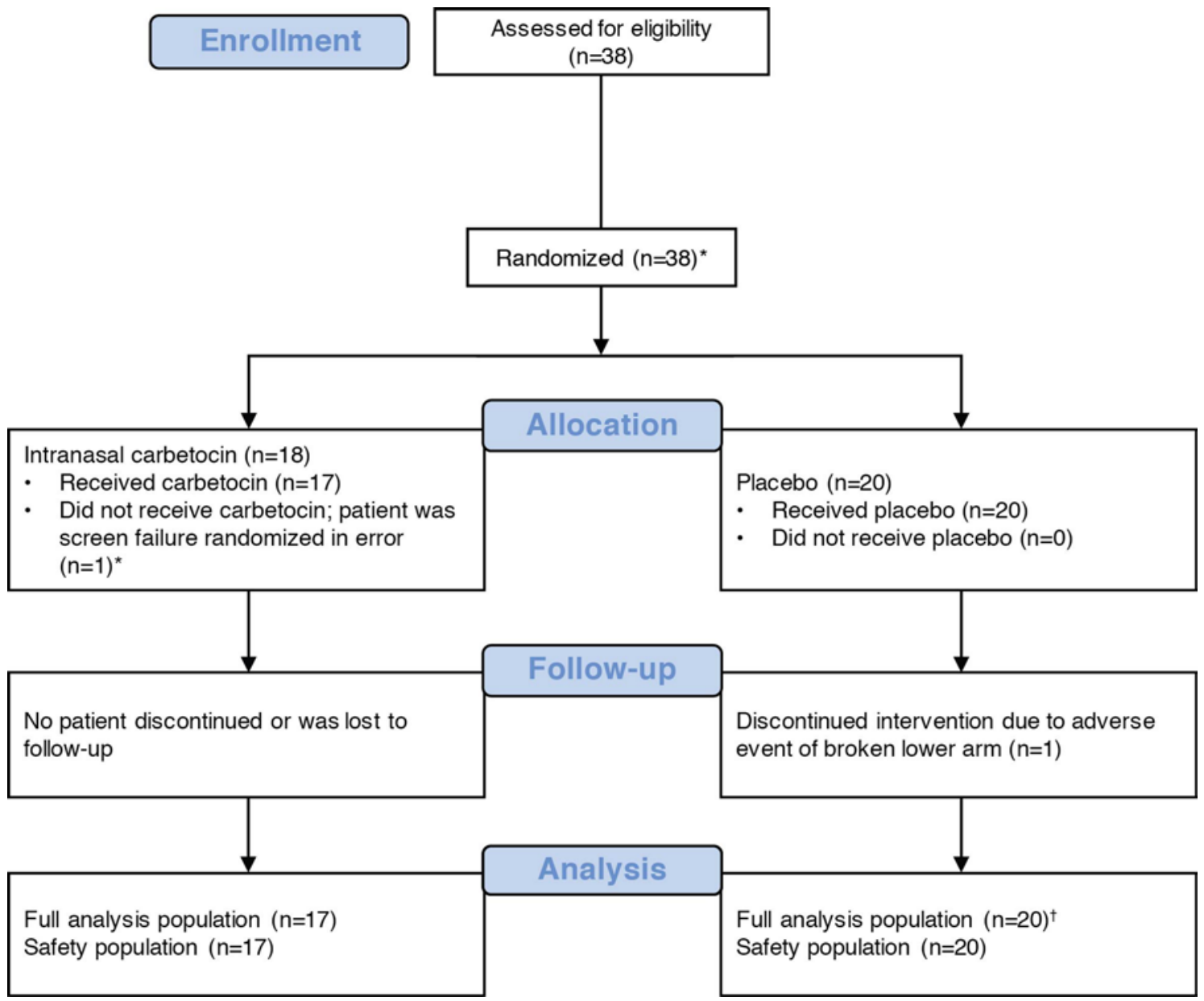

Figure 1. CONSORT diagram of disposition of study patients. * One patient who did not meet inclusion criteria was mistakenly randomized to treatment with carbetocin but did not receive any study medication. ${ }^{\dagger} A$ total of 19 patients in the placebo group completed the study; however, 1 patient who discontinued the study because of an AE (broken lower arm) was included in the full analysis population and participated in the final efficacy assessment on day 15 .

(5). Patients with PWS also manifest significant compulsive behaviors, including insistence on sameness, repetitive questioning, rearranging items until they are "just right," skin picking, and hoarding (6). Additionally, patients are prone to impulsivity, anxiety, tantrums, and sudden outbursts, with some variability in the expression of all of these based on environmental factors, age, and genetic subtypes of PWS (6).

The combination of hyperphagia, intellectual disabilities, and behavioral difficulties presents life-long management challenges for parents and families, and it limits the range of social, educational, and vocational opportunities for individuals with PWS. Parents and caregivers are tasked with exerting some level of environmental controls, including close supervision of children around food, securing food sources (e.g., locking the refrigerator), providing reduced caloric meals and snacks, adhering to a predictable meal schedule to ameliorate anxiety, encouraging regular exercise, and advocating for their child's dietary needs in educational and other settings (7). While strict environmental controls may help reduce caloric intake and effectively manage weight for some individuals with PWS in the home setting, the persistent hyperphagic drive and associated behavioral problems characteristic of PWS often prevent patients from achieving a high quality of life or being well-integrated into their communities.

While complications of obesity are common causes of death in PWS, related causes of morbidity are observed regardless of body mass index (BMI) and include gastric necrosis and rupture after an episode of binge eating (8), as well as choking due to sneaking food and eating too rapidly while unsupervised (9). The clinical benefits of treating hyperphagia with weight-loss drugs used in the general population, psychotropic medications (10), and restrictive bariatric surgeries have not been established (11). No pharmacotherapies are currently available to effectively reduce symptoms of hyperphagia in PWS. However, the potential benefit of 
Table 1. Baseline demographics and clinical characteristics of study participants - full analysis set

\begin{tabular}{|c|c|c|c|}
\hline Demographics & Carbetocin $(n=17)$ & Placebo $(n=20)$ & Overall $(n=37)$ \\
\hline Male, $n(\%)$ & $6(35.3)$ & $8(40.0)$ & $14(37.8)$ \\
\hline \multicolumn{4}{|l|}{ Age, years } \\
\hline Mean (SD) & $13.9(2.4)$ & $13.6(2.5)$ & $13.7(2.5)$ \\
\hline Range & $10.0-18.0$ & $10.0-18.0$ & $10.0-18.0$ \\
\hline White/European descent & $17(100)$ & $19(95)$ & $36(97.3)$ \\
\hline \multicolumn{4}{|l|}{ Weight, kg } \\
\hline Mean (SD) & $66.3(17.2)$ & $61.82(20.7)$ & $63.89(19.1)$ \\
\hline \multicolumn{4}{|l|}{ Height, cm } \\
\hline Mean (SD) & $160.3(10.0)$ & $154.32(10.3)$ & $157.0(10.4)$ \\
\hline UPD & 7 & 6 & 13 \\
\hline
\end{tabular}

i.n. delivery of an oxytocin analog in patients with PWS may be gleaned from existing evidence of the specific neurologic actions of this hormone. Indeed, oxytocin is a potent anorexigenic hormone, and it is integral to the complex neural and gut networks associated with the homeostatic control of food intake, satiety, and energy balance (12). The proform of oxytocin is cleaved by subtilisin-like prohormone convertase (PC1), an enzyme that is encoded by the proprotein convertase subtilisin/kexin type 1 gene (PCSK1) (13).

Recent evidence indicates that deficits in PC1 (resulting from paternal deletion of the noncoding RNA gene SNORD116) may contribute to the development of clinical phenotypes observed in patients with PWS due to improper processing of hormones like oxytocin (14). Moreover, findings of postmortem studies in individuals with PWS demonstrate a lower number of oxytocin-secreting neurons in the paraventricular nucleus of the hypothalamus (15), suggesting that dysregulation of oxytocin impairs the satiety response in patients with PWS.

Several studies have investigated the effects of i.n. oxytocin treatment in patients with PWS and have produced conflicting results. Tauber and colleagues (16) conducted a randomized, double-blinded, placebo-controlled study of a single 24-IU dose of i.n. oxytocin in 24 adult inpatients with PWS. Two days after administration, the oxytocin group had less sadness tendencies (irritability, frustration), less destructive behaviors (tantrums, aggression), and more trust in others (greeting people, joining activities) compared with the placebo group. Although these outcomes were based on unstandardized staff ratings, they are consistent with a growing animal and human literature on the role of oxytocin in pair bonding, empathy, attachment security, and trust (17-19). Miller and colleagues (20) recently reported results of a randomized, double-blinded, placebo-controlled, 2-period crossover study designed to evaluate i.n. oxytocin in 24 children aged 5-11 years with PWS. After 5 days of treatment, i.n. oxytocin 16 IU was superior to placebo in all 17 behavioral, socialization, anxiety, and appetite endpoints, although none achieved statistical significance.

In contrast, Einfeld and colleagues (21) found no beneficial effects of i.n.oxytocin in a double-blinded, placebo-controlled trial in 30 children and adolescents with PWS. Using dosages that varied from 18-40 IU twice daily for 8 weeks, investigators found no treatment effects in hyperphagia, compulsivity, or social functioning. Instead, a significant worsening of temper outbursts was reported for patients receiving higher doses of oxytocin. These observations may be explained by oxytocin's lack of specificity and stimulation of arginine vasopressin (AVP) receptors, which are not reduced in the PWS brain. Vasopressin differs from oxytocin by just 2 amino acids and has been associated with states of negative emotional arousal and aggression (22). Thus, high doses of i.n. oxytocin may bind to the more abundant AVP receptors and 


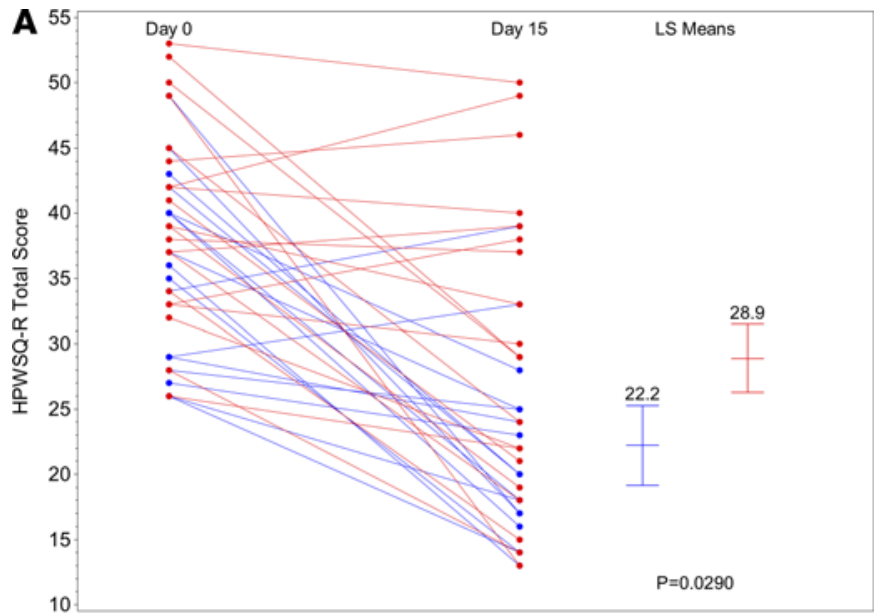

Planned Treatment Carbetocin - Placebo

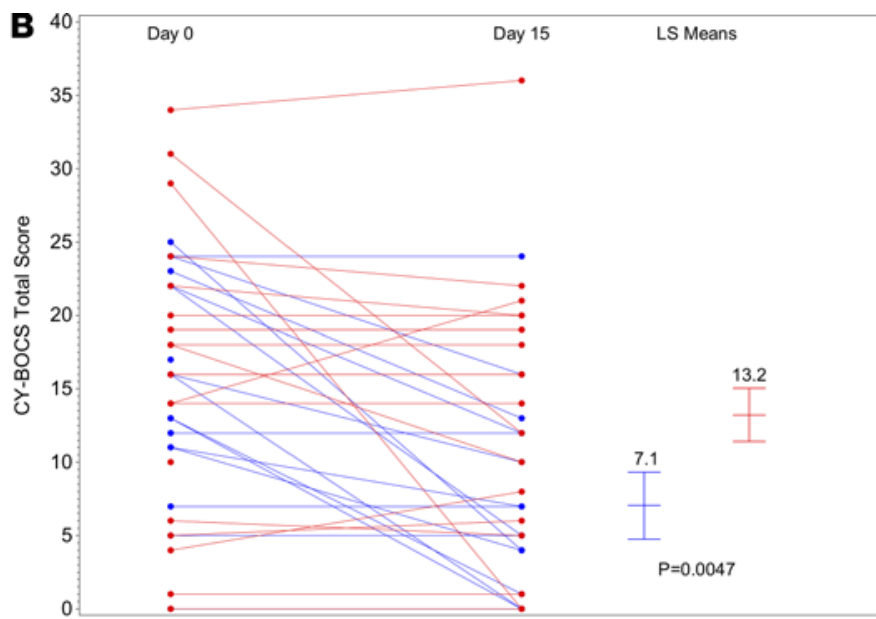

Planned Treatment Carbetocin - Placebo

Figure 2. Change from baseline in HPWSQ-R and CY-BOCS at study end (visit 4, day 15). (A) HPWSQ-R total scores at baseline and study end (visit 4, day 15; primary efficacy endpoint) and least squares (LS) mean change versus baseline for each treatment group and $90 \%$ Cls. (B) CY-BOCS total scores at baseline and study end (visit 4, day 15; secondary efficacy endpoint) and LS mean change versus baseline for each treatment group and $90 \%$ Cls. Statistical analyses included all participants with assessment scores at baseline and day 15; analyses were performed using an ANCOVA model, with treatment group and study site as fixed effects. Baseline HPWSQ-R total scores served as a covariate. Treatment group differences in total scores were calculated by subtracting LS mean change from baseline in the placebo group from that in the carbetocin group. HPWSQ-R, parent/caregiver-rated Hyperphagia in PWS Questionnaire-Responsiveness.

worsen behavioral symptoms (22). In addition, oxytocin carries the risk of prolonged antidiuresis (reduced urinary volume) and hyponatremia (low sodium blood levels) owing to considerable vasopressin V2 receptor activity $(23,24)$. Therefore, an oxytocin receptor-selective compound may be preferable to avoid these vasopressin-related medical complications or potential worsening of agitated behavior. Indeed, some individuals with PWS are known to have polydipsia (excessive thirst) and low baseline serum sodium levels (25). As such, antidiuretic effects from exogenous oxytocin therapy have the potential to further increase the risk of the serious complication of hyponatremia.

Carbetocin is an analog of the endogenous hormone oxytocin with an improved receptor-selectivity profile. It is approved in numerous countries outside the US for the prevention of uterine atony and

Table 2. Change from baseline in secondary efficacy endpoints at study end (visit 4, day 15)

\begin{tabular}{|c|c|c|c|c|}
\hline & $\begin{array}{l}\text { Carbetocin } \\
(n=17)\end{array}$ & $\begin{array}{l}\text { Placebo } \\
(n=20)\end{array}$ & \multirow[t]{2}{*}{ Adjusted mean difference } & \multirow[t]{2}{*}{$P$ value (1-sided) } \\
\hline Efficacy outcome & \multicolumn{2}{|c|}{ LS mean (SE) score } & & \\
\hline HPWSQ-R & & & & \\
\hline Behavior domain & $-5.9(1.6)$ & $-3.9(1.2)$ & -2.0 & 0.117 \\
\hline Drive domain & $-4.5(1.5)$ & $-2.9(1.2)$ & -1.6 & 0.143 \\
\hline Severity domain & $-3.3(0.8)$ & $-1.8(0.6)$ & -1.5 & 0.025 \\
\hline Behavior domain & $-7.7(1.7)$ & $-3.4(1.3)$ & -4.3 & 0.007 \\
\hline Drive domain & $-5.7(1.4)$ & $-3.2(1.1)$ & -2.6 & 0.034 \\
\hline Severity domain & $-4.0(0.7)$ & $-1.8(0.6)$ & -2.2 & 0.001 \\
\hline CGI-I & $2.7(0.5)$ & $3.6(0.4)$ & -0.8 & 0.023 \\
\hline CY-BOCS & $-8.8(2.3)$ & $-2.6(1.9)$ & -6.2 & 0.005 \\
\hline
\end{tabular}

Efficacy endpoints were analyzed statistically using an ANCOVA model with treatment and site as fixed effects and baseline scale scores as covariates. Adjusted mean difference indicates difference between least square (factors included) means. CGI-I, Clinical Global Impressions-Improvement; CY-BOCS, Children's Yale-Brown Obsessive-Compulsive Scale; HPWSQ-R, parent/caregiver-rated Hyperphagia in Prader-Willi Syndrome QuestionnaireResponsiveness; HPWSQ-C, clinician-rated Hyperphagia in Prader-Willi Syndrome Questionnaire; LS, least squares; SE, standard error. 
Table 3. TEAEs with incidence $\geq 5 \%$ in either treatment group, safety population, $n(\%)$

\begin{tabular}{lccc}
\hline TEAEs by preferred term & Carbetocin $(\boldsymbol{n}=\mathbf{1 7})$ & Placebo $(\boldsymbol{n}=\mathbf{2 0})$ & Overall ( $\mathbf{n}=\mathbf{3 7})$ \\
Any TEAE & $7(41.2)$ & $8(40.0)$ & $15(40.5)$ \\
Headache & $5(29.4)$ & $6(30.0)$ & $1(29.7)$ \\
Medication error & $1(5.9)$ & $2(10.0)$ & $3(8.1)$ \\
Abdominal pain upper & $1(5.9)$ & $1(5.0)$ & $2(5.4)$ \\
Conjunctivitis infective & $1(5.9)$ & $0(0)$ & $1(2.7)$ \\
Diarrhea & $1(5.9)$ & $0(0)$ & $1(2.7)$ \\
Dysgeusia & $1(5.9)$ & $1(5.0)$ & $1(2.7)$ \\
Aggression & $0(0)$ & $1(5.0)$ & $1(2.7)$ \\
Hyperphagia & $0(0)$ & $1(5.0)$ & $1(2.7)$ \\
Procedural pain & $0(0)$ & $1(5.0)$ & $1(2.7)$ \\
Sinusitis & $0(0)$ & $1(5.0)$ & $1(2.7)$ \\
Ulna fracture & $0(0)$ & $1(5.0)$ & $1(2.7)$ \\
Urine analysis abnormal & $0(0)$ & $1(2.7)$
\end{tabular}

TEAE, treatment-emergent adverse event.

excessive bleeding during cesarean section delivery. Carbetocin has a well-established safety profile, with an estimated cumulative exposure of 9.5 million patients in 80 countries outside the US. The rationale for conducting the current phase 2 trial was, thus, based on (a) the improved oxytocin receptor-selectivity profile of carbetocin; (b) established safety in humans; and (c) converging evidence of the role of oxytocin in human behaviors that are aberrant in PWS (12). As such, the objective of the current study was to assess the efficacy and safety of i.n. carbetocin as a targeted treatment for symptoms of hyperphagia in patients with PWS.

\section{Results}

Patient disposition and demographics. Of the 38 patients randomly assigned to receive treatment, 1 patient in the carbetocin group was excluded from the study because of prior or concomitant use of prohibited medication and did not receive treatment. A total of 37 patients received treatment with carbetocin or placebo and were included in full analysis and safety populations (Figure 1). One patient in the placebo group prematurely discontinued the study because of an adverse event (AE; ulnar fracture); the remaining 36 patients completed the trial. Demographics and baseline characteristics, including BMI, were similar between the 2 treatment groups, although patients in the carbetocin group were slightly taller and heavier than those in the placebo group (Table 1).

Efficacy endpoints. In the primary efficacy endpoint analysis, patients who received carbetocin had statistically significant reductions from baseline in the parent/caregiver-rated Hyperphagia in PWS Questionnaire-Responsiveness (HPWSQ-R) total score at end of treatment (least squares [LS] mean change: -15.6) compared with those who received placebo $(-8.9 ; P=0.029$; Figure $2 \mathrm{~A})$.

Results of secondary efficacy endpoint analyses are summarized in Table 2 and Supplemental Figures 1-4 (supplemental material available online with this article; https://doi.org/10.1172/jci. insight.98333DS1). Statistically significant improvements were observed for patients who received carbetocin versus those who received placebo for secondary endpoint LS mean changes from baseline in the HPWSQ-R severity domain score $(P=0.025)$; clinician-rated HPWSQ (HPWSQ-C) total score and behavior, drive, and severity domain scores $(P \leq 0.034)$; Children's Yale-Brown Obsessive Compulsive Scale (CY-BOCS; $P=0.005$; Figure $2 \mathrm{~B}$ ); and food domain of the Reiss Profile scores $(P=0.01)$. In addition, Clinical Global Impressions-Improvement (CGI-I) total scores indicated a statistically significant treatment effect of carbetocin at study end $(P=0.023)$. Highly statistically significant correlations were found between the primary endpoint change in HPWSQ-R total score and all supportive secondary endpoints including change in HPWSQ-C total score, change in CY-BOCS total score, change in Reiss Profile food domain, and CGI-I score $(r=0.60-0.93 ; P \leq 0.0001)$, indicating that treatment-related improvements in hyperphagia were associated with overall symptom improvement. Supplemental Table 1 provides the mean change from baseline in primary and secondary efficacy outcome measures at days 8 
and 15 for the full analysis population. In the comparison of CY-BOCS and CGI-I results, 10 patients in the carbetocin group had a CGI-I rating of 1 (very much improved) or 2 (much improved), and all had a $\geq 35 \%$ reduction in CY-BOCS scores. In contrast, only 1 patient in the placebo group had a CGI-I of 1 or 2 and a $\geq 35 \%$ reduction in CY-BOCS score, demonstrating a statistically significant difference between treatment groups $(P<0.001)$.

Safety assessments. The incidence of treatment-emergent AEs (TEAEs) occurring in $\geq 5 \%$ of either treatment group was evenly distributed across the carbetocin and placebo groups (Table 3). In the carbetocin group, 7 patients (41.2\%) reported 26 TEAEs, with a majority (96\%) categorized as mild; 1 TEAE was moderate. In the placebo group, 8 (40\%) patients reported 29 TEAEs, with $86 \%$ categorized as mild; 4 TEAEs were moderate. One patient in the placebo group was withdrawn from the study because of moderate TEAEs that included agitation, increased aggression, and hyperphagia related to a broken distal ulnar. The most common TEAE was headache, reported in 5 patients who received carbetocin and 6 who received placebo (overall 29.7\%). No severe TEAEs were observed during the study.

No notable changes from baseline were observed in vital sign values, physical examination findings, ECG tracings, or laboratory values for patients in the carbetocin or placebo groups. Upon physical examination, no moderate or clinically significant signs of nasal irritation were reported during the study. All nasal examinations were graded as "no abnormal findings," "focal nasal mucosal inflammation," or "superficial nasal mucosal erosion." A total of 4 of 17 patients in the carbetocin group and 3 of 20 patients in the placebo group who had "no abnormal findings" at baseline had "focal nasal mucosal inflammation" at study end; no changes from baseline were observed in the remaining patients.

\section{Discussion}

This is the first randomized, placebo-controlled trial to demonstrate significant improvement in hyperphagia and related behavioral problems during treatment with the therapeutic peptide carbetocin in patients with PWS. Compared with placebo, a 14-day regimen of i.n. carbetocin significantly reduced hyperphagic symptoms, food-related behaviors and emotions, and compulsivity, while also improving overall functioning. Responses were rapid and consistent with the expected pharmacology of i.n. carbetocin (unpublished data on file). Treatment with i.n. carbetocin demonstrated a favorable safety profile, with no safety issues identified during the study. Nasal irritation was mild and infrequent, and the only AE resulting in study withdrawal was distress related to a broken lower arm for 1 patient in the placebo group. I.n. carbetocin was, thus, well tolerated throughout the 14 days of treatment. The beneficial effects of carbetocin identified in the current study contrast with a previous study of i.n. oxytocin in patients with PWS published by Einfeld and colleagues, which showed no positive effects and demonstrated a worsening of aggressive behavior in response to i.n. oxytocin (21). Such differences may be attributed to the improved oxytocin receptor-selectivity profile of carbetocin.

As studies assessing the efficacy of pharmacotherapies for hyperphagia in PWS are limited, the HPWSQ was benchmarked against 2 widely used outcome measures in clinical trials, the CGI-I and CY-BOCS. Changes from baseline in HPWSQ, CGI-I, and CY-BOCS scores were highly correlated, and treatment-related improvements in all secondary measures support the clinical meaningfulness of improvements observed on the primary efficacy endpoint. Such converging evidence suggests that a HPWSQ treatment effect of this magnitude may be an indicator of clinically meaningful change in future clinical trials.

The degree of control over access to food in the patients' home environments was not collected or assessed. The extent to which these commonly instituted controls impact HPWSQ values and sensitivity to treatment is unknown. However, if a family is diligent about locking trash in the home, for example, one would expect that the likelihood that they report their child foraging through the trash at any point during the study would be reduced. Therefore, it would be difficult to assess changes in this item of the HPWSQ.

Interestingly, clinicians generally reported more robust improvements in hyperphagic symptoms than parents in the current study. There is a renewed research emphasis on patient-reported outcomes, yet less guidance exists on how to best interpret data when parents serve as a proxy for offspring with cognitive deficits or limited insight. Parents are uniquely positioned to observe changes in their children with PWS, yet because they are "in the trenches" providing daily care and management, they may inadvertently accommodate to their child's symptoms. Family accommodation is significant in treatment studies of pediatric patients with obsessive-compulsive or other anxiety disorders $(26,27)$. One of many desirable outcomes of a treatment option for PWS would include the ability for families to relax preexisting rigid environmental 
controls. However, the duration of treatment required to sufficiently convince caregivers and patients that these controls are less necessary is unknown and is likely longer than 2 weeks. Future clinical trials in PWS or other developmental disabilities need to carefully consider how to best balance ratings from parents and/ or clinicians as indices of treatment outcome.

Although this phase 2 safety and efficacy trial used a robust design, it also had some noteworthy limitations. First, power was not sufficient to evaluate possible interactions between genetic subtypes of PWS and treatment response to carbetocin. Importantly, while those with paternal deletions versus maternal uniparental disomy may differ in specific psychiatric vulnerabilities, these subgroups show, on average, very similar levels of hyperphagia (7). Second, as this was a proof-of-concept study designed to assess the safety and efficacy of a potentially novel agent in a medically complex developmental disorder, an inherent limitation was the treatment period of 14 days. This short trial duration precluded assessments regarding the impact of hyperphagia reduction with carbetocin on social or emotional functioning; such assessments would require longer-term treatment. Moreover, although repetitive, compulsive behaviors were assessed in the current study, more fine-tuned analyses are necessary to identify possible differential effects of carbetocin on specific compulsive symptoms. The short trial duration also limited assessments of the effects of i.n. carbetocin on weight and BMI in patients with PWS. Interestingly, the overall patient population had a mean BMI value of $25.7 \mathrm{~kg} / \mathrm{m}^{2}$ at baseline (range, $15.8-40.0 \mathrm{~kg} / \mathrm{m}^{2}$ ), an observation that further supports the notion that environmental restrictions routinely set forth by parents/caregivers may help in preventing obesity. Importantly, the goal of the study was not to reduce BMI but instead curb the hyperphagic behavior, drive, and severity that contribute to obesity in this population. Indeed, individuals with PWS and normal BMI still experience hyperphagic symptoms and other behavioral disturbances and, as previously noted, are at high risk for medical complications related to binging (gastric rupture, necrosis) or food sneaking (choking). Finally, study patients all received the same dose of carbetocin, and it is not known if similar treatment effects can be achieved under different dosing regimens. Despite these limitations, to our knowledge, this is the first randomized, placebo-controlled trial to demonstrate significant and clinically meaningful improvements in patients with PWS following a limited period of treatment with i.n. carbetocin. The long-term effects of carbetocin in PWS remain unknown, and findings from this study set the stage for larger trials of longer duration. A full development program to evaluate the safety and efficacy of carbetocin for the treatment of PWS, sponsored by Levo Therapeutics Inc., is currently underway.

Conclusions. In this study, i.n. carbetocin significantly reduced hyperphagic symptoms compared with placebo and exhibited a favorable safety profile in patients with PWS. Treatment with carbetocin over 14 days reduced compulsivity and improved overall functioning. Larger trials with longer treatment duration are needed to further assess the efficacy of i.n. carbetocin on hyperphagia, compulsivity, and social and emotional functioning, as well as to determine if improvements in behavior complement weight reduction in individuals with PWS. As effective treatments for PWS stand to have an enormous impact on families and affected individuals, future research should consider how carbetocin or other treatments of hyperphagic behavior may reduce caregiver stress and burden, improve quality of life for patients with PWS and their families, and allow patients to be more fully engaged with their families, peers, and communities.

\section{Methods}

\section{Study participants}

Eligible study patients were aged 10-18 years, had genetically confirmed PWS (as reported by caregivers who provided written reports of previous genetic subtype testing), and exhibited hyperphagia, as confirmed by being in PWS nutritional phase 3 (hyperphagia) (5) and by having a baseline HPWSQ (7) total score $>13$ at screening. Exclusion criteria were a concurrent diagnosis of autism spectrum disorder, active psychosis, major surgery within 6 months and/or nasal or sinus surgery within 1 year, chronic sinusitis (more than 3 episodes per year), cardiovascular disorders, epilepsy, frequent migraines, severe asthma, or low serum sodium levels ( $<135 \mathrm{mmol} / \mathrm{l})$. Prior or concomitant use of growth hormone therapy was permitted, as was the use of psychotropic medications or thyroid hormone replacement therapy, as long as the prescribed dosage of medications was stable for $\geq 6$ months. Products containing prostaglandins and i.n. therapies (including nasal saline) were prohibited. No information about the food environment where the participants lived was collected. 


\section{Study design and treatment}

This prospective, randomized, double-blind, placebo-controlled parallel trial (ClinicalTrials.gov: NCT01968187) was conducted at 3 US sites from January 20, 2014, to July 16, 2014. Patients were randomly assigned (1:1) to receive carbetocin or placebo (sterile sodium chloride $0.9 \%)$. An unblinded statistician from $\mathrm{H}_{2} \mathrm{O}$ Clinical prepared a computer-generated list of randomization numbers using SAS version 9.2 or higher (SAS Institute Inc.) with a block size of 4.

Randomization numbers were assigned sequentially in a blinded manner to patients according to their chronological entry into the study using an interactive Web response system. An onsite pharmacist was provided treatment allocation envelopes that appeared in numeric order, and once a patient's eligibility was confirmed, the pharmacist selected the next randomization number. The randomization list was not available to any clinical trial personnel, other than an onsite pharmacist, until the trial database was released to the statistician.

I.n. doses were administered 3 times daily for 14 days, prior to meals, and according to prespecified time intervals (morning dose: 6:00 to 9:00 a.m.; midday dose: 11:00 a.m. to 1:00 p.m.; evening dose: 4:30 to 6:00 p.m.). A single dose consisted of 3 spray-pump actuations per nostril (each spray pump delivering $50 \mu 1$ volume of solution).

Patients in the active treatment group received $9.6 \mathrm{mg}$ of carbetocin per dose. The dose and dosing regimen were based on results from a previous phase 1 trial (unpublished data on file).

Parents/caregivers and patients visited the investigational site 4 times over the course of the trial and participated in 2 assessments via telephone interview. Screening visit evaluations for eligibility included a physical examination, vital signs, height, weight, 12-lead ECG, nasal examination, laboratory assessments, and administration of the HPWSQ (7). Blood samples for clinical laboratory and pharmacokinetic evaluation were performed at selected visits. Study visit 2 (day 1) included administration of the first treatment dose, parent training on i.n. administration procedures, and efficacy assessments. Participants were again dosed during study visit 3 (day 2), with clinical supervision of parent administration of study medication. On day 8, parents participated in a phone interview assessment, and on day 15 , parents and patients returned to the study site for final evaluations (study visit 4). A follow-up phone call was made to patients after the study ended (day $19 \pm 3$ ).

\section{Study assessments}

Primary efficacy endpoint. The primary efficacy endpoint was LS mean change from baseline in the HPWSQ-R total score at end of treatment (study visit 4). The HPWSQ-R is a parent/caregiver-rated measure that identifies the behavioral and psychological features of hyperphagia in PWS (7). The HPWSQ-R was adapted from the original hyperphagia questionnaire for use in phenotypic studies of PWS and as a responsiveness measure for intervention or treatment trials. Items are rated on a 5-point Likert scale and are summed for a total score, with higher scores indexing more hyperphagic symptoms. Previous factor analyses identified 3 robust domains, accounting for $58.93 \%$ of variance (7), including hyperphagic behavior (e.g., manipulating others to obtain food, sneaking food), drive (e.g., ease in redirecting away from food, distress when denied food), and severity (e.g., extent that hyperphagia interferes with daily routines, time spent asking or talking about food). In the current trial, parents or caregivers completed the HPWSQ-R using a 1-week recall period. The baseline Cronbach's $\alpha$ for HPWSQ-R was 0.85 , indicating good internal consistency of items.

Secondary efficacy endpoints. Analyses of secondary endpoints included change from baseline to end of treatment (study visit 4 ) in the 3 domain scores (i.e., behavior, drive, and severity) on the HPWSQ-R, and changes in total and domain scores on the HPWSQ-C, which consisted of the same 11 questions as on the HPWSQ-R, based on the same 1-week recall period. The baseline Cronbach's $\alpha$ for the HPWSQ-C was 0.80 , which is consistent with the HPWSQ-R and other studies using parents as informants (7). Additional secondary efficacy measures included change in total scores on the CY-BOCS (28), food domain of the Reiss Profile (29) rated by the parent/caregiver, and CGI-I (30) score at the end of treatment. The CY-BOCS is a clinician-rated, semistructured inventory of the types and severity of obsessions and compulsions that characterize pediatric obsessive-compulsive and related disorders; the scale is widely used and has robust psychometric properties, including sensitivity to change in treatment studies $(28,31-33)$. Total scores on the CY-BOCS are calculated using a symptom checklist and severity scale. The food domain of the Reiss Profile (29) consists of 7 questions that assess food-related behaviors and motivation (e.g., "eating is more important [for participant] than for others," "enjoys eating more than others," "often asks about 
the next meal"). Items are rated on a 5-point scale ranging from -2 ("strongly disagree") to 2 ("strongly agree"). The CGI-I is a 7-point clinician-administered instrument (1, very much improved since baseline/ initiation of treatment; 7, very much worse from baseline) that takes into account the patient's history, psychosocial circumstances, and behavior, as well as the impact of symptoms on the participant's overall ability to function; this scale is commonly used to assess treatment response $(30,34)$.

Safety. Safety assessments were based on a review of all reported AEs, TEAEs, laboratory findings, vital signs, ECG tracings, physical exam findings, and nasal mucosal assessments. Nasal examinations were performed at baseline, day 8 , and day 15 using a prespecified grading system.

\section{Statistics}

The primary efficacy analysis was change from baseline to the end of treatment on day 15 in the HPWSQ-R total score. With 30 participants eligible for the primary efficacy analysis, the study had $\geq 80 \%$ power to detect a statistically significant difference (at the 1-sided 10\% significance level) between the i.n. carbetocin and placebo groups, assuming the true treatment group difference was at least -5 . At the time of the trial design, the available literature suggested that the HPWSQ-R total score is normally distributed with a standard deviation of 6 (7). Assuming a dropout rate of up to $20 \%, 38$ randomized participants were needed to ensure that at least 30 participants would be eligible for the primary efficacy analysis.

The primary efficacy analysis included all participants who had an HPWSQ-R assessment at baseline, day 8 , and/or day 15, and was performed using an analysis of covariance (ANCOVA) model with treatment group and study site as fixed effects. One-sided $P$ values of 0.01 were specified for continued development of the drug; $P<0.05$ were considered statistically significant.

Baseline HPWSQ-R total scores served as a covariate for consistency with European Medicines Agency guidelines (35). The treatment group difference in HPWSQ-R total score between placebo and carbetocin was calculated by subtracting the LS mean change from baseline in the placebo group from that in the carbetocin group. A statistically significant difference at the $10 \%$ significance level would be achieved if the upper limit of the 90\% 1-sided CI for the treatment difference was less than zero. All secondary efficacy endpoints were analyzed in a similar manner using the same ANCOVA model. Pearson correlations were calculated between end-of-treatment change from baseline scores on the HPWSQ-R primary endpoint and end-of-treatment change from baseline scores on other secondary endpoints assessed. To further explore secondary endpoints, a comparison was made between the CY-BOCS and CGI-I based on results from Farris and colleagues (36). These authors reported that a $\geq 35 \%$ reduction on the Y-BOCS was optimal in predicting treatment response, defined by CGI-I ranks of 1 ("very much improved") or 2 ("much improved"). All statistical analyses were prespecified prior to unblinding the study data.

\section{Study approval}

Prior to enrollment, parents/caregivers and patients with PWS received verbal and written information about the trial. An IRB for each study site (Vanderbilt Human Research Protection Program Health Sciences Committee \#3, IRB00002125; Western IRB, Puyallup, Washington, USA, IRB00000533; and Winthrop University Hospital IRB, FWA00000726) reviewed and approved this trial protocol and amendments, informed consent forms, and materials provided to subjects. The trial was conducted in accordance with the Declaration of Helsinki and in compliance with the International Conference on Harmonization Guidelines for Good Clinical Practice. Consistent with IRB-approved procedures, parents/caregivers provided written informed consent, and patients with PWS provided informed assent. Parents were trained on the proper use of the nasal spray device and demonstrated their ability to do so prior to leaving the study site.

\section{Author contributions}

EMD, JM, MA, ER, MR, HTH, RW, GB, and PK contributed study design. EMD, JM, ER, MR, HTH, RW, GB, and PK acquired, analyzed, and interpreted data. EMD, JM, MA, ER, MR, HTH, RW, GB, and $\mathrm{PK}$ drafted and revised the manuscript for content. Final approval for publication was conducted by EMD, JM, MA, ER, MR, HTH, RW, GB, and PK.

\section{Acknowledgments}

The authors thank the families and individuals with PWS who participated in this study, as well as Christine Keeling, study coordinator; Stephen Couch, the lead clinical investigator at Vanderbilt University; and 
Hailee Hunt-Hawkins and Nathan Dankner at Vanderbilt University for their assistance in data collection. Levo Pharmaceuticals Inc. currently holds the rights for carbetocin development in PWS. However, Ferring Pharmaceuticals Inc. funded this study at the time it was conducted. Editorial assistance was provided by Peloton Advantage LLC and funded by Ferring Pharmaceuticals Inc. Recruitment for the current study was aided by ongoing work in PWS performed through a Eunice Kennedy Shriver National Institute of Child Health and Human Development grant U54 HD083211.

Address correspondence to: Michael Reidy,Ferring Pharmaceuticals Inc., 100 Interpace Parkway, Parsippany, New Jersey 07054, USA. Phone: 973.796.4442; Email: michael.reidy@ferring.com.

RW's present address is: Insmed Inc., Bridgewater, New Jersey, USA.

PK's present address is: Korner BioPharm Advisors LLC, Franklin Lakes, New Jersey, USA.

1. Lionti T, Reid SM, White SM, Rowell MM. A population-based profile of 160 Australians with Prader-Willi syndrome: trends in diagnosis, birth prevalence and birth characteristics. Am J Med Genet A. 2015;167A(2):371-378.

2. Angulo MA, Butler MG, Cataletto ME. Prader-Willi syndrome: a review of clinical, genetic, and endocrine findings. $J$ Endocrinol Invest. 2015;38(12):1249-1263.

3. Jin DK. Systematic review of the clinical and genetic aspects of Prader-Willi syndrome. Korean J Pediatr. $2011 ; 54(2): 55-63$.

4. Cassidy SB, Driscoll DJ. Prader-Willi syndrome. Eur J Hum Genet. 2009;17(1):3-13.

5. Miller JL, et al. Nutritional phases in Prader-Willi syndrome. Am J Med Genet A. 2011;155A(5):1040-1049.

6. Dykens EM, Roof E. Behavior in Prader-Willi syndrome: relationship to genetic subtypes and age. J Child Psychol Psychiatry. 2008;49(9):1001-1008.

7. Dykens EM, Maxwell MA, Pantino E, Kossler R, Roof E. Assessment of hyperphagia in Prader-Willi syndrome. Obesity (Silver Spring). 2007;15(7):1816-1826.

8. Stevenson DA, et al. Gastric rupture and necrosis in Prader-Willi syndrome. J Pediatr Gastroenterol Nutr. 2007;45(2):272-274.

9. Stevenson DA, et al. Deaths due to choking in Prader-Willi syndrome. Am J Med Genet A. 2007;143A(5):484-487.

10. Dykens E, Shah B. Psychiatric disorders in Prader-Willi syndrome: epidemiology and management. CNS Drugs. 2003;17(3):167-178.

11. Scheimann AO, Butler MG, Gourash L, Cuffari C, Klish W. Critical analysis of bariatric procedures in Prader-Willi syndrome. J Pediatr Gastroenterol Nutr. 2008;46(1):80-83.

12. Sabatier N, Leng G, Menzies J. Oxytocin, feeding, and satiety. Front Endocrinol (Lausanne). 2013;4:35.

13. Pan H, et al. Neuropeptide processing profile in mice lacking prohormone convertase-1. Biochemistry. 2005;44(12):4939-4948.

14. Burnett LC, et al. Deficiency in prohormone convertase PC1 impairs prohormone processing in Prader-Willi syndrome. J Clin Invest. 2017;127(1):293-305.

15. Swaab DF, Purba JS, Hofman MA. Alterations in the hypothalamic paraventricular nucleus and its oxytocin neurons (putative satiety cells) in Prader-Willi syndrome: a study of five cases. J Clin Endocrinol Metab. 1995;80(2):573-579.

16. Tauber M, et al. Oxytocin may be useful to increase trust in others and decrease disruptive behaviours in patients with Prader-Willi syndrome: a randomised placebo-controlled trial in 24 patients. Orphanet J Rare Dis. 2011;6:47.

17. Bartz JA, et al. Oxytocin selectively improves empathic accuracy. Psychol Sci. 2010;21(10):1426-1428.

18. Buchheim A, et al. Oxytocin enhances the experience of attachment security. Psychoneuroendocrinology. 2009;34(9):1417-1422.

19. Kosfeld M, Heinrichs M, Zak PJ, Fischbacher U, Fehr E. Oxytocin increases trust in humans. Nature. 2005;435(7042):673-676.

20. Miller JL, et al. Oxytocin treatment in children with Prader-Willi syndrome: A double-blind, placebo-controlled, crossover study. Am J Med Genet A. 2017;173(5):1243-1250.

21. Einfeld SL, et al. A double-blind randomized controlled trial of oxytocin nasal spray in Prader Willi syndrome. Am J Med Genet A. 2014;164A(9):2232-2239.

22. Thompson RR, George K, Walton JC, Orr SP, Benson J. Sex-specific influences of vasopressin on human social communication. Proc Natl Acad Sci USA. 2006;103(20):7889-7894.

23. Seifer DB, Sandberg EC, Ueland K, Sladen RN. Water intoxication and hyponatremic encephalopathy from the use of an oxytocin nasal spray. A case report. J Reprod Med. 1985;30(3):225-228.

24. Ansseau M, et al. Intranasal oxytocin in obsessive-compulsive disorder. Psychoneuroendocrinology. 1987;12(3):231-236.

25. Akefeldt A. Water intake and risk of hyponatraemia in Prader-Willi syndrome. J Intellect Disabil Res. 2009;53(6):521-528.

26. Strauss C, Hale L, Stobie B. A meta-analytic review of the relationship between family accommodation and OCD symptom severity. J Anxiety Disord. 2015;33:95-102.

27. Insel TR. The challenge of translation in social neuroscience: a review of oxytocin, vasopressin, and affiliative behavior. Neuron 2010;65(6):768-779.

28. Scahill L, et al. Children's Yale-Brown Obsessive Compulsive Scale: reliability and validity. J Am Acad Child Adolesc Psychiatry. 1997;36(6):844-852.

29. Havercamp SM, Reiss S. A comprehensive assessment of human strivings: test-retest reliability and validity of the Reiss Profile. J Pers Assess. 2003;81(2):123-132.

30. Guy W. ECDEU Assessment Manual for Psychopharmacology Revised, 1976. The Internet Archive. https://archive.org/ details/ecdeuassessmentm1933guyw. Accessed May 18, 2018

31. Storch EA, et al. Psychometric evaluation of the Children's Yale-Brown Obsessive-Compulsive Scale. Psychiatry Res. 
2004;129(1):91-98.

32. Yucelen AG, Rodopman-Arman A, Topcuoglu V, Yazgan MY, Fisek G. Interrater reliability and clinical efficacy of Children's Yale-Brown Obsessive-Compulsive Scale in an outpatient setting. Compr Psychiatry. 2006;47(1):48-53.

33. Pediatric OCD Treatment Study (POTS) Team. Cognitive-behavior therapy, sertraline, and their combination for children and adolescents with obsessive-compulsive disorder: the Pediatric OCD Treatment Study (POTS) randomized controlled trial. JAMA. 2004;292(16):1969-1976.

34. Busner J, Targum SD. The clinical global impressions scale: applying a research tool in clinical practice. Psychiatry (Edgmont). 2007;4(7):28-37.

35. European Medicines Agency. Guideline on adjustment for baseline covariates in clinical trials. 2015. Available at: http://www. ema.europa.eu/docs/en_GB/document_library/Scientific_guideline/2015/03/WC500184923.pdf. Accessed: October 18, 2017.

36. Farris SG, McLean CP, Van Meter PE, Simpson HB, Foa EB. Treatment response, symptom remission, and wellness in obsessive-compulsive disorder. J Clin Psychiatry. 2013;74(7):685-690. 